\title{
Genetic Engineering of Transgenic Tobacco for Enhanced Uptake and Bioaccumulation of Mercury
}

\author{
Takeshi Nagata, Asako Nakamura, Toshifumi Akizawa, and Hidemitsu Pan-Hou* \\ Faculty of Pharmaceutical Sciences, Setsunan University; 45-1 Nagaotogecho, Hirakata, Osaka 573-0101, Japan. \\ Received March 17, 2009; accepted May 19, 2009; published online June 23, 2009
}

To further enhance the efficiency and potential of plants for phytoremediation of mercury pollution, a genetically engineered tobacco to simultaneously express mercury transporter, mercury transporter (MerT) and mercury chelator, polyphosphate (polyP) was constructed by integrating bacterial merT gene in polyphosphate kinase gene (ppk)-transgenic tobacco, and its ability to phytoremediate mercury was evaluated. Integration of $m e r T$ gene into $p p k$-transgenic tobacco did not significantly affect the mercury resistant phenotypes and polyP production. Transgenic expression of MerT in ppk-transgenic tobacco resulted in accelerated and enhanced mercury uptake into tobacco. In addition, tobacco expressing MerT and polyP accumulated significantly more mercury than the ppk-transgenic tobacco from medium containing a wide range of low concentrations of $\mathrm{Hg}^{2+}$. $\mathrm{The}^{-}$ combination of accelerated mercury uptake and enhanced mercury accumulation mediated by MerT represents one way for shortening the purification completion time, and for improving tobacco plants to be more suitable for use in phytoremediation of low levels of mercury contamination.

Key words mercury transporter; polyphosphate kinase gene; mercury; uptake; accumulation; phytoremediation

Mercury contamination in water and soil often jeopardizes the ecosystem stability and poses a serious danger to human health so an effective and affordable technological solution is needed. Current methodologies for remediation of mercurypolluted soils mainly rely on excavation and landfilling of the polluted sites. Many sites remain contaminated with no remediation in sight simply because it is too expensive and labor-intensive to clean up with the available technologies.

Phytoremediation, using green plants to extract and/or sequester contaminants may provide an economically viable solution for cleaning up some of these contaminated sites. Recently, with advances in biotechnology, phytoremediation utilizing specific plants to remove mercurials from contaminated sites has become one of the most rapidly developing fields for environmental restoration. Several studies have successfully integrated bacterial mercury-resistant (mer) genes into plant genome to create superior plants for phytoremediation of mercury-contaminated sites, based on the merA-mediated mercury reduction and volatilization mechanism. ${ }^{1-5}$ However, phytovolatilization of mercurials mediated by bacterial merA gene from the polluted sites would still cause public anxiety because of the release into the ambient air of mercury vapor that will redistribute in the ecosphere and be recycled into the environment.

The ideal plants for phytoremediation process should not only be able to efficiently tolerate and bioaccumulate high levels of particular contaminants in its harvestable parts but also have the potential to produce a high biomass in the field. ${ }^{6-8)}$ In previous papers, we reported that expression of polyphosphate (polyP) encoded by bacterial polyphosphate kinase gene $(p p k)$ in a genetically engineered tobacco resulted in significantly increased accumulation of $\mathrm{Hg}^{2+}$ from simulated soils without releasing mercury vapor into the ambient, and protected the tobacco from the toxicity of the accumulated $\left.\mathrm{Hg}^{2+} .{ }^{9}, 10\right)$ These phenotypes in addition to their high biomass production, the engineered tobacco should satisfy the proposed prerequisites for plants used in the phytoremediation of mercury in soils.

In general, rehabilitation of metal-contaminated soils by plants requires a long time for the purification process to be completed. McGrath and Zhao ${ }^{11)}$ reported that several months were required to reduce the metal content by half in contaminated soils. The expression of metal transporter in the plant may provide a means of improving metal uptake, thereby shortening the purification completion time. In this study, a genetically engineered tobacco was constructed to simultaneously express mercury transporter, MerT, and overexpress mercury accumulator, polyP. The ability of the transgenic tobacco's ability to remediate mercury was also investigated. Here, we report that transgenic expression of MerT in tobacco resulted not only in accelerated mercury uptake but also in enhanced mercury accumulation. The combination of accelerated mercury uptake and enhanced mercury accumulation may be a particularly promising strategy for shortening the completion time, and for improving tobacco plants so they become more suitable for phytoremediation of low-level mercury contamination.

\section{MATERIALS AND METHODS}

Materials and Growth Conditions Escherichia coli XL1-Blue was grown at $37^{\circ} \mathrm{C}$ in Luria-Bertani (LB) medium $^{12)}$ and used for routine plasmid propagation. Wildtype tobacco (Nicotiana tabacum cv. Samsun NN), kindly supplied by Japan Tobacco and the $\mathrm{T}_{3}$ progeny of $p p k$-transgenic tobacco, engineered to express bacterial $p p k$-specified polyphosphate ${ }^{9)}$ were photoautotrophically cultured in Murashige-Skoog (MS) medium ${ }^{13)}$ according to the method of Takeda et al. (1990). ${ }^{14)}$ Agrobacterium tumefaciens LBA4404 and binary vector $\mathrm{pHM6}$, a $\mathrm{pBin} 19$ derivative ${ }^{15)}$ was kindly provided by Prof. K. Yazaki of Kyoto University.

Plasmid Construction and Agrobacterium Transformation The $0.4 \mathrm{~kb}$ merT gene (GeneBank/DDBJ accession No. D83080) of Pseudomonas K-62 was amplified by polymerase chain reaction (PCR) using two specific primers. The sense primer, designated as ATU (5'-GCTCTAGACTAGATCTAAGAAGGAACCACAATGTCTGAACCACAAAAC$\left.3^{\prime}\right)$ contained a $X b a$ I cloning site, a consensus Shine-Dal- 
garno (SD) bacterial ribosome binding site (AGAAGG), a consensus sequence for plant translation (PT) (AACCACA), a start codon (ATG) and the first 15-nt of the merT coding sequence to prime the forward PCR reaction. The antisense primer, designated as AML (5'-GCTCTAGAGGTTACAAGTCCTCTTCAGAAATCAGCTTTTGCTCATAGAAAAATGGAACGAC-3'), contained a XbaI cloning site, anticodons to 30-nt of human $c$-myc epitope tag codon (5'-GAGCAAAAGCTGATTTCTGAAG AGGACTTG-3') and anticodons to the last 7-nt merT codon to prime the reverse PCR reaction. PCR was carried out for 30 cycles with denaturing, annealing, and extending temperature/time of $94^{\circ} \mathrm{C}$ for $1 \mathrm{~min}, 60^{\circ} \mathrm{C}$ for $1.5 \mathrm{~min}$, and $68^{\circ} \mathrm{C}$ for $2 \mathrm{~min}$. The amplified merT::myc fusion fragment $(0.4-\mathrm{kb})$ was subcloned into cloning vector Bluescript II SK(+) to confirm the nucleotide sequence and then cleaved in the flanking $X b a \mathrm{I}$ site, and the resulting fragment was subcloned into the binary expression vector pHM6, downstream of the E12 promoter that consists of Cauliflower mosaic virus $35 \mathrm{~S}$ promoter having two enhancers in tandem and an omega sequence to create plant expression plasmid, pT2C77 (Fig. 1).

Plant Transformation and Regeneration The pT2C77 was transformed into Agrobacterium tumefaciens LBA4404 by electroporation and its sequence was confirmed. The $p p k$ transgenic tobacco (T3) was transformed with this $A$. tumefaciens, and transformants were selected by $30 \mu \mathrm{g} / \mathrm{ml}$ hygromycin and regenerated according to previously published procedures. ${ }^{9)}$ Healthy seedlings of $4-6 \mathrm{~cm}$ in height were transferred to the MS medium with antibiotic to initiate root growth. Following root induction, the plantlets were transferred onto soil and grown to maturity. $\mathrm{T}_{2}$ plants were used for all experiments described in this paper.

Detection of Transgene Genomic DNA was isolated from transgenic and control tobacco leaves using a DNeasy Plant Mini kit (Qiagen, Valencia, CA, U.S.A.). The PCR primer pair, ATU and AML was used to confirm the integration of merT::myc gene into tobacco. PCR analysis was performed using a PCR Thermal Cycler (TaKaRa, Kyoto, Japan) according to previously published procedures. ${ }^{9)}$

Western-Blot Analysis Extraction of total soluble proteins from tobacco leaves and immunoblotting using the monoclonal anti-human c-Myc antibody was carried out according to the standard protocol reported by Nakano et al. ${ }^{16)}$ and the manufacturer's instructions, respectively.

Mercury Tolerance Assay The sterilized seeds from wilt-type, $p p k$-transgenic and merT/ppk-transgenic tobacco were germinated and grown in MS agar medium containing various concentrations of $\mathrm{Hg}^{2+}$. After 2 weeks incubation at $25^{\circ} \mathrm{C}$, mercury tolerance was evaluated by monitoring the fresh weight according to the method previously described. ${ }^{9,10)}$

Mercury Uptake and Accumulation Assay The 4 weeks-old seedlings were hydroponically grown in fresh MS liquid medium containing $0.75 \mu \mathrm{M} \mathrm{Hg}^{2+}$. After incubation for appropriate times at $25^{\circ} \mathrm{C}$, mercury taken up into the tobacco leaves was determined by flameless cold-vapor atomic adsorption spectrometry using an atomic mercury analyzer (RA-2A, Nippon Instruments, Tokyo, Japan) after the samples were digested with concentrated nitric acid. ${ }^{17)}$ Mercury accumulation in tobacco leaves was measured as described previously.,10) Briefly, the sterilized seeds from ppk-trans-

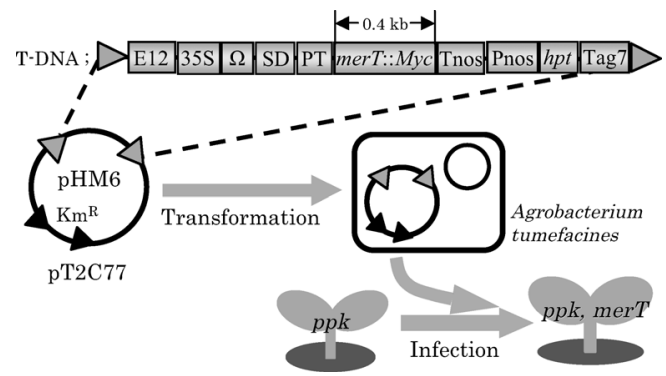

Fig. 1. Experimental Design for Engineering of merT in $p p k$-Transgenic Tobacco

The PCR amplified merT::myc fragment $(0.4-\mathrm{kb})$ was subcloned into the binary expression vector pHM6, downstream of the E12 promoter that consists of Cauliflower mosaic virus $35 \mathrm{~S}$ promoter having two enhancers in tandem and an omega sequence to create a plasmid pT2C77. The transferred DNA (t-DNA) region in pT2C77 was transformed into ppk-transgenic tobacco via Agrobacterium mediated gene transfer as described in Materials and Methods. Tnos; nopaline synthase terminator, Pnos; nopaline synthase promoter, hyt; hygromysin phosphotransferase, Tag7; agropine terminator. $\mathrm{SD}$; bacterial ribosome binding site, PT; plant translation signal.

genic and $m e r T / p p k$-transgenic tobacco were germinated and grown in MS agar medium containing various concentrations of $\mathrm{Hg}^{2+}$. After 2 weeks incubation at $25^{\circ} \mathrm{C}$, the leaves of tobacco were collected and the amount of mercury in the leaves was determined.

\section{RESULTS}

Expression of merT Gene in ppk/merT-Transgenic Tobacco The bacterial mercury transport gene, merT from Pseudomonas K-62 fused with human $c-m y c$ epitope tag, was modified for plant expression and transferred into $p p k$-transgenic tobacco using Agrobacterium (Fig. 1). After selection by $30 \mu \mathrm{g} / \mathrm{ml}$ hygromycin and regeneration, a large number of positive independent $\mathrm{ppk} / \mathrm{merT}$-transgenic shoots were obtained. No considerable morphological abnormalities were observed in all the transformants, which grew, flowered and set seeds vigorously at rates similar to non-transformed $p p k$ transgenic tobacco (data not shown).

To confirm the existence of merT::myc gene in the $p p k / m e r T$-transgenic tobacco, total DNA from the mature leaves of $\mathrm{T}_{2}$ progeny was extracted, and genomic DNA-PCR analysis was performed. As expected, $0.4-\mathrm{kb}$ product was detected in $p p k / m e r T$-transgenic tobacco but not in the wildtype and $p p k$-transgenic tobacco when ATU and AML were used as PCR primers (Fig. 2A). By Western blot analysis of the extracts from mature leaves, a novel protein band of $15 \mathrm{kDa}$ in accordance with the expected molecular weight of the MerT::Myc protein, which reacted specifically with antihuman c-Myc antibody, was detected in $p p k / m e r T$-transgenic tobacco, but not in the wild-type and $p p k$-transgenic tobacco (Fig. 2B).

Effect of MerT on Mercury Tolerance of $p p k / m e r T$ Transgenic Tobacco The effect of expression of MerT on the mercury tolerance of transgenic tobacco was evaluated by monitoring the fresh weight of shoots after 2 weeks exposure to various concentrations of $\mathrm{Hg}^{2+}$ at $25^{\circ} \mathrm{C}$. In agreement with our previous results, ${ }^{9,10)}$ the $p p k$-transgenic tobacco showed more resistance to $\mathrm{Hg}^{2+}$ than its wild-type progenitors (Fig. 3). Introduction of merT gene into ppk-transgenic tobacco did not significantly affect the mercury-resistant phenotype shown by the ppk-transgenic tobacco (Fig. 3). 
A

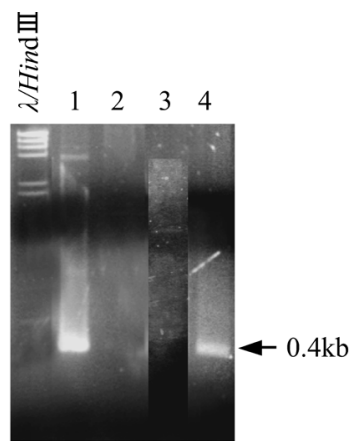

B

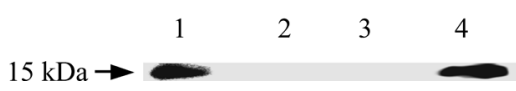

Fig. 2. PCR Analysis of merT Gene (A) and Western Blot Analysis of MerT Protein (B) in Transgenic Tobacco

Genome DNA was extracted from leaves of wild-type (Lane 2), ppk-transgenic (Lane 3 ) and $m e r T / p p k$-transgenic (Lane 4) tobacco. The PCR was performed using two pairs of merT-myc-construct specific oligoprimer (ATU/AML) as described in Materials and Methods. The positive DNA control (Lane 1) is the pT2C77 construct PCR product. Crude protein extracted from leaves of wild-type (Lane 2), ppk-transgenic (Lane 3) and merT/ppk-transgenic (Lane 4) tobacco were probed with anti-Myc monoclonal antibody as described in Materials and Methods. Arrow indicates purified MerT-Myc protein (Lane 1) isolated from E. coli expressing a recombinant merT-myc gene.

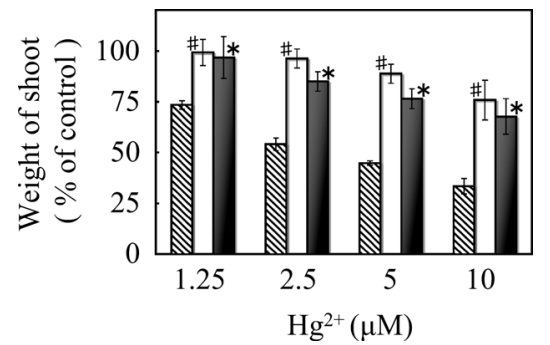

Fig. 3. Mercury Sensitivity

The sterilized seeds from wild-type $(\mathbf{\nabla}), p p k$-transgenic $(\square)$ and merT/ppk-transgenic ( $\mathbf{\square})$ tobaccos were germinated and grown on MS agar medium containing various concentrations of $\mathrm{Hg}^{2+}$. After 2 weeks, the culture sensitivity of tobacco to $\mathrm{Hg}^{2+}$ was evaluated by monitoring the fresh weight of shoots as described in Materials an Methods. Data are expressed as mean \pm S.E.M. of three determinations from 3 independent experiments. $* p<0.05 v s$. wild-type tobacco. ${ }^{2} p<0.05 v s$. wild-type tobacco.

Effect of MerT on Mercury Uptake and Mercury Accumulation Uptake of mercury by $p p k / m e r T$-transgenic tobacco was determined after hydroponic culture in MS medium containing $0.75 \mu \mathrm{M} \mathrm{Hg}^{2+}$ at $25^{\circ} \mathrm{C}$. As shown in Fig. 4 , the $p p k / m e r T$-transgenic tobacco took up approximately 2 fold more mercury than the $p p k$-transgenic tobacco from $\mathrm{Hg}^{2+}$-containing medium. The rate of $\mathrm{Hg}^{2+}$ uptake was significantly accelerated by the expression of MerT in the transgenic tobacco (Fig. 4).

Mercury accumulation in transgenic tobacco leaves was determined after 2 weeks exposure to various concentrations of $\mathrm{Hg}^{2+}$ in MS agar medium. As shown in Fig. 5, the ppk/ merT-transgenic tobacco accumulated significantly more mercury from medium containing $0.1-1.25 \mu \mathrm{M} \mathrm{Hg}^{2+}$ than did the ppk-transgenic tobacco. However, there was no significant difference between the mercury accumulations of the two transgenic tobaccos at $\mathrm{Hg}^{2+}$ concentrations above 2.5 $\mu \mathrm{M}$.

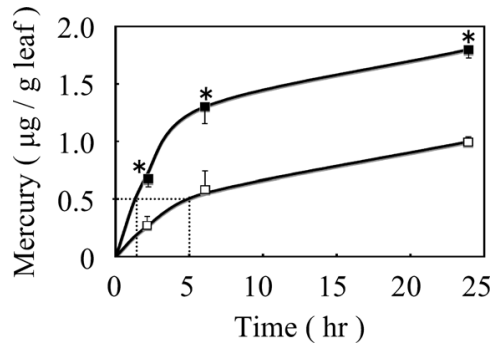

Fig. 4. Mercury Uptake

Sterilized seeds of $p p k$-transgenic $(\square)$ and merT/ppk-transgenic ( $\square$ ) tobacco were germinated and grown in MS agar medium for 3 weeks at $25^{\circ} \mathrm{C}$. The seedlings were transferred to the MS liquid medium containing $0.75 \mu \mathrm{M} \mathrm{Hg}^{2+}$. After incubation for the appropriate length of times, total mercury in the tobacco leaves was determined. Data are expressed as mean \pm S.E.M. of three determinations from 3 independent experiments. $* p<0.05$ vs. $p p k$-transgenic tobacco.
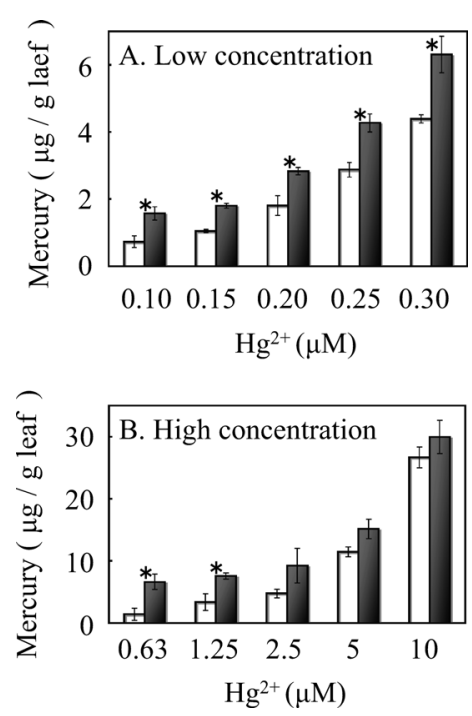

Fig. 5. Mercury Accumulation

Total mercury in tobacco leaves of $p p k$-transgenic $(\square)$ and merT/ppk-transgenic ( $\square$ ) tobacco was determined after 2 weeks culture at $25^{\circ} \mathrm{C}$ with various concentrations of $\mathrm{Hg}^{2+}$ (A: Low concentration, B: High concentration) as described in Materials and Methods. Data are expressed as mean \pm S.E.M. of three determinations from 3 independent experiments. $* p<0.05 v s$. ppk-transgenic tobacco.

\section{DISCUSSION}

Decontamination of mercury in soil has long been a challenge. Phytoremediation, using green plants to remove environmental pollutants including hazardous toxic metals removal from a large volume of contaminated sites is recognized as a cost-effective, sustainable and aesthetically pleasing technology. However, the use of plants, like all biological methods, does not allow $100 \%$ removal of contaminants because the remediation rates decrease as the concentrations of contaminant decrease. In addition, phytoremediation is a slow process that requires a long time to complete the purification. Therefore, for contaminated site that poses acute risks for human and other ecological receptors, phytoremediation may not be the remediation technique of choice. These potential faults may predominantly result from the low metaluptake activity and thereby limit its usefulness for practical application.

Among the strategies being used to overcome these disadvantages is the use of metal transporter to boost the uptake and transport of metal from soil into transgenic plants. Ex- 
pression of heavy metal transporter genes under the control of a constitutive or inducible promoter would be attractive for phytoremediation. Hirschi et al. ${ }^{18)}$ reported that transfer of the calcium transporter CAX-2 from A. thaliana to tobacco resulted in enhanced accumulation of $\mathrm{Ca}, \mathrm{Cd}$ and $\mathrm{Mn}$. Transgenic Arabidopsis overexpressing yeast protein YCF1, a member of $\mathrm{ABC}$ transporter family involved in transfer of $\mathrm{Cd}$, accumulated more $\mathrm{Cd}$ and $\mathrm{Pb}$ than the wild-type control plants. ${ }^{19)}$ In the present study, the effect of the mercury transporter, MerT on the effectiveness and efficiency of mercury phytoremediation was evaluated.

By using Agrobacterium-mediated gene transfer, a large number of putative, independent transgenic tobaccos resistant to hygromycin were obtained. In some of these plants, it was confirmed by PCR analysis that merT::myc gene was stably integrated in the ppk-transformed tobacco (Fig. 2A). Among them, a novel extra protein band at $15 \mathrm{kDa}$, thought to be the MerT::Myc fusion protein, was identified by sodium dodecyl sulfate-polyacrylamide gel electrophoresis (SDS-PAGE) and confirmed by Western blotting (Fig. 2B). These results clearly demonstrated that the merT::myc fusion gene was successfully integrated into the $p p k$-transgenic tobacco and substantially translated to the expected MerT:: Myc protein in the transgenic tobacco. By specific staining with 4',6'-diamidino-2-phenylindole, a large amount of polyP was also detected around the leaf vein of $\mathrm{ppk} / \mathrm{merT}$ transgenic tobacco (data not shown), suggesting that integration of merT gene did not have a deleterious effect on the biosynthesis of polyP encoded by ppk gene in the ppk/merTtransgenic tobacco. In addition, integration of merT gene into $p p k$-transgenic tobacco did not significantly affect the mercury resistant phenotype (Fig. 3). These results suggest that the incorporated mercury from medium was converted to a less toxic molecule, probably via chelation with $p p k$-specified polyP in the transgenic tobacco tissue as previously described. ${ }^{21,22)}$ These characteristics in addition to the ability to overexpress mercury chelator, poly $\mathrm{P}^{9,10)}$ shown by the $p p k /$ merT-transgenic tobacco may fulfill the proposed prerequisites for plants used for phytoremediation of mercury in soils.

Using a hydroponic culture system, tobacco plants expressing MerT took up mercury at enhanced levels compared to the uptake by control ppk-transgenic tobacco (Fig. 4). The rate of $\mathrm{Hg}^{2+}$ uptake appeared to be $c a$. two-times higher in $p p k / m e r T$-transgenic tobacco than in control $p p k$-transgenic tobacco. These results reveal that MerT is indeed functional as a mercury transporter in transgenic tobacco plants, and suggest that accelerated mercury uptake into tobacco mediated by MerT would provide one possible way for shortening the completion time of phytoremediation of mercury pollution.

Phytoremediation of mercury is a complex process, involving uptake into the plant, translocation into the shoot, and resistance against and accumulation of this toxic metal in the plants. When present at elevated levels in soil, mercurials are able to enter tobacco plants via a passive diffusion mechanism or through essential metal transport systems by virtue of their chemical similarities to other metals. ${ }^{20)}$ Previously, we reported that tobacco plants expressing polyP in the absence of MerT, accumulated 2-6-fold more mercury than its wild-type progenitors from soils containing $\mathrm{Hg}^{2+}$ concentra- tions over a range of $2.5-10 \mu \mathrm{M}$, but did not accumulate appreciable amounts of $\mathrm{Hg}^{2+}$ compared with the wild-type control from soils containing $\mathrm{Hg}^{2+}$ concentrations below $1.25 \mu \mathrm{M}$, despite its possession of polyP. ${ }^{9,10)}$ These previous observations together with the fact that mercury accumulation was accelerated by expression of MerT in tobacco plants inspired us to ascertain the efficiency and potential of ppk/merT-transgenic tobacco for use in the phytoremediation of low levels of mercury contamination, which is generally believed to be difficult to cleanup using natural or genetically-engineered plants without transport systems for the pollutant. Tobacco engineered to express mercury transporter and polyP accumulates effectively $\mathrm{Hg}^{2+}$ at a range of low concentrations of $\mathrm{Hg}^{2+}(0.1-1.25 \mu \mathrm{M})$ (Fig. 5). However, at relatively high concentrations of $\mathrm{Hg}^{2+}$ (above $2.5 \mu \mathrm{M}$ ), there was no significant difference between the mercury accumulation of the two tobacco plants, $p p k$ and $p p k / m e r T$. A simple interpretation for this incongruity is that mercury uptake by passive diffusion is predominant over the uptake mediated by MerT. At present, we do not have enough information to warrant further discussion, and thus more detailed studies that include identification of the cellular localization of MerT, are required to achieve a more complete understanding of this incongruity. The results obtained here demonstrate that expression of mercury transporter, MerT into ppk-transgenic tobacco represents one way for improving plants to be more suitable for phytoremediation of low levels of mercury present in polluted sites.

Phytoremediation is an effective and aesthetically pleasing technique for cleaning up soils contaminated with mercurials where excavation or bioremediation is not practical or possible. However, the technique is still in its infancy stage. Our results demonstrate that the use of genetic engineering to modify plants to express the mercury transporter, MerT, may open up new avenues for enhancing the efficiency of phytoremediation of mercury in polluted soils. It is hoped that, in the near future, data we are obtaining in artificial laboratory experiments can be validated in field experiment and help the design of new strategies.

Acknowledgments We are grateful to Dr. M. Kiyono of Kitasato University for her technical assistance in the cloning pT2C77 and constructive suggestions concerning the manuscript. We also thank M. Ando, Y. Takemura and Y. Okazaki of this University for their technical assistance. The wildtype tobacco (Nicotiana tabacum cv. Samsun NN) was a generous gift from the Japan Tobacco Inc. This work was supported in part by a Grant-in-Aid to H.H. from the Ministry of the Environment of Japan.

\section{REFERENCES}

1) Bizily S. P., Pugh C. L., Meagher R. B., Nat. Biotechnol., 18, 213 217 (2000).

2) Bizily S. P., Kim T., Kandasamy M. K., Meagher R. B., Plant Physiol., 131, 463-471 (2003).

3) Rugh C. L., Wilde H. D., Stack N. M., Thompson D. M., Summers A. O., Meagher R. B., Proc. Natl. Acad. Sci. U.S.A., 93, 3182-3187 (1996).

4) Rugh C. L., Senecoff J. F., Meagher R. B., Merkle S. A., Nat. Biotechnol., 16, 925-928 (1998).

5) Ruiz O. N., Hussein H. S., Terry N., Daniell H., Plant Physiol., 132, 1344-1352 (2003) 
6) Meagher R. B., Curr. Opin. Plant Biol., 3, 153-162 (2000).

7) Lasat M. M., J. Environ. Qual., 31, 109-120 (2002).

8) McGrath S. P., Zhao F. J., Lombi E., Adv. Agron., 75, 1-56 (2002).

9) Nagata T., Kiyono M., Pan-Hou H., Appl. Microbiol. Biotechnol., 72, 777-782 (2006).

10) Nagata T., Kiyono M., Pan-Hou H., Biol. Pharm. Bull., 29, 23502353 (2006).

11) McGrath S. P., Zhao F. J., Curr. Opin. Biotechnol., 14, 277-282 (2003).

12) Sambrook J., Fritsch E. F., Maniatis T., "A Laboratory Manual," 2nd ed., Cold Spring Harbor Laboratory, NY, 1989.

13) Murashige T., Skoog F., Physiol. Plant, 15, 473-449 (1962).

14) Takeda S., Sato F., Ida K., Yamada Y., Plant Cell Physiol., 31, 215221 (1990).
15) Gatz C., Frohberg C., Wendenburg R., Plant J., 40, 397-404 (1992).

16) Nakano T., Murakami S., Shoji T., Yoshida S., Yamada Y., Sato F., The Plant Cell, 27, 1673-1682 (1997).

17) Pan-Hou H., Hosono M., Imura N., Appl. Environ. Microbiol., 40, 1007-1011 (1980).

18) Hirschi K. D., Korenkov V. D., Wilganowski N. L., Wagner G. J., Plant Physiol., 124, 125-133 (2000).

19) Song W. Y., Sohn E. J., Martinoia E., Lee Y. J., Jasinski M., Forestier C., Hwang I., Lee Y., Nat. Biotechnol., 21, 914-919 (2003).

20) Hall J. L., Williams L. E., J. Exp. Bot., 54, 2601-2613 (2003).

21) Pan-Hou H., Kiyono M., Kawase T., Omura T., Endo G., Biol. Pharm. Bull., 24, 1423-1426 (2001).

22) Pan-Hou H., Kiyono M., Omura H., Omura T., Endo G., FEMS Microbiol. Lett., 207, 159-164 (2002). 